\title{
Phenylketonuria screening in Iranian newborns: a systematic review and meta- analysis
}

Mehdi Shokri ${ }^{1}$, Parviz Karimi ${ }^{1}$, Hadis Zamanifar ${ }^{2}$, Fatemeh Kazemi ${ }^{3}$, Gholamreza Badfar $^{4}$ and Milad Azami ${ }^{*}$ (D)

\begin{abstract}
Background: Phenylketonuria (PKU), which is characterized by a deficiency of phenylalanine hydroxylase activity, is an autosomal recessive disorder of phenylalanine (Phe) metabolism. Newborn screening is the main populationbased public health screening program that allows successful identification and treatment of PKU with low-Phe diet. The aim of this study was to evaluate the epidemiology of PKU screening in Iranian newborns.
\end{abstract}

Methods: The present study was designed based on MOOSE protocol and reporting was done in accordance with the PRISMA guidelines. The protocol of this systematic review was published in PROSPERO before it was performed (CRD42020162626). A comprehensive search was done in 10/10/2019 to find related literature on international online databases Web of Science, Scopus, EMBASE, Science Direct, PubMed/Medline, EBSCO, CINAHL, Cochrane Library, national online databases and the Google Scholar search engine. Heterogeneity among studies was assessed by $\mathrm{I}^{2}$ index and $\mathrm{Q}$ test. All meta-analyses were performed using Comprehensive Meta-Analysis Software ver. 2. $P<0.05$ was considered significant.

Result: Finally, 18 studies with 3,339,327 Iranian neonates were included. The prevalence of suspected hyperphenylalaninemia (HPA) was estimated to be 45.6/100,000 (95\% Cl: 23.9-87.1). The prevalence of suspected HPA in girls and boys infants in Iran was estimated to be 38.0/100,000 (95\% Cl: 15.1-95.5) and 43.3/100,000 (95\% Cl: 16.2-116.2), respectively. The prevalence of PKU was estimated to be $16.5 / 100,000$ (95\% Cl: 12.9-21.2). The prevalence of PKU in girls and boys infants was estimated to be 13.3/100,000 (95\% Cl: 7.5-15.8) and 10.9/100,000 (95\% Cl: 7.5-15.8), respectively. The prevalence of mild to moderate HPA was estimated 9.7/100,000 (95\% Cl: $5.1-$ 18.4) and the prevalence of classical PKU was estimated 4.4/100,000 (95\% Cl: 2.5-7.8). Sensitivity analysis for all meta-analysis with the omission of one study showed that overall estimation is still robust.

Conclusion: The results of this meta-analysis showed that PKU is prevalent in Iranian neonates. It should be considered that for PKU there is a highly effective dietary treatment which can prevent the clinical symptoms of PKU if initiated early after detection by newborn screening.

Keywords: Phenylketonuria, Iran, Newborn, Meta-analysis

\footnotetext{
* Correspondence: milad98azami@gmail.com

${ }^{5}$ School of Medicine, llam University of Medical Sciences, llam, Iran

Full list of author information is available at the end of the article

(C) The Author(s). 2020 Open Access This article is licensed under a Creative Commons Attribution 4.0 International License, which permits use, sharing, adaptation, distribution and reproduction in any medium or format, as long as you give appropriate credit to the original author(s) and the source, provide a link to the Creative Commons licence, and indicate if changes were made. The images or other third party material in this article are included in the article's Creative Commons licence, unless indicated otherwise in a credit line to the material. If material is not included in the article's Creative Commons licence and your intended use is not permitted by statutory regulation or exceeds the permitted use, you will need to obtain permission directly from the copyright holder. To view a copy of this licence, visit http://creativecommons.org/licenses/by/4.0/ The Creative Commons Public Domain Dedication waiver (http://creativecommons.org/publicdomain/zero/1.0/) applies to the data made available in this article, unless otherwise stated in a credit line to the data.
} 


\section{Background}

Phenylketonuria (PKU), which is characterized by a deficiency of phenylalanine hydroxylase (PAH) activity, is an autosomal recessive disorder of phenylalanine (Phe) metabolism [1]. If left untreated, high blood Phe concentrations pass through the blood brain barrier and cause harmful effects on the growth and function of brain [2].

The main treatment for PKU is low Phe diet. It is recommended that treatment be started as soon as possible and continue throughout life. Although a restricted diet is beneficial for PKU patients, long-term adherence to diet is a difficult challenge, especially for teenagers and those preparing for pregnancy [3].

If urine tyrosine and tetrahydrobiopterin levels are normal and Phe levels are above $20 \mathrm{mg} / \mathrm{dl}$, between 10 and $20 \mathrm{mg} / \mathrm{dl}$, and between 2 and $10 \mathrm{mg} / \mathrm{dl}$, newborns are diagnosed with severe or classical PKU, mild PKU and hyperphenylalaninemia (HPA), respectively [4].

Nowadays, clinical manifestations of classical PKU are rarely reported in developed countries, where newborn screening (NBS) is common. NBS is the main population-based public health screening program that allows successful identification and treatment of PKU with low-Phe diet. Early dietary treatment leads to normal results for patients with this disorder [5]. The first NBS program appeared in the United States in the early 1960s [6] and then spread to most developed countries [7]. PKU can be easily detected in heel prick test $24 \mathrm{~h}$ after birth using novel diagnostic methods [8]. There are various methods for detecting PKU in dried blood spot (DBS) sampling, such as fluorometric and colorimetric methods [9], enzymatic method [10, 11], highperformance liquid chromatographic (HPLC) [10], and new methods such as Tandem Mass Spectrometry $[12,13]$.

Numerous studies have shown that the prevalence of PKU is inconsistent in different Iranian studies and is still a challenging issue [14-31]. Meta-analysis is a statistical method for combining the data of multiple studies with the same goal. When the effect size is consistent between studies, meta-analysis can be used to identify this common effect. Finally, meta-analysis results can provide a more accurate estimate of the impact of treatment or risk factors for disease or other outcomes by combining individual studies [32-34]. The aim of this study was to evaluate the epidemiology of PKU screening in Iranian newborns.

\section{Method}

\section{Study protocol}

The present study was designed based on Meta-analyses Of Observational Studies in Epidemiology (MOOSE) protocol [35] and reporting was done in accordance with the Preferred Reporting Items for Systematic Reviews and Meta-analysis (PRISMA) guidelines [34]. Given the type of study, the approval of the Ethics Committee was not required. All study phases were performed independently by two authors. In cases where there were disagreements, they were resolved through group discussion. The protocol of this systematic review was published in PROSPERO before it was performed (CRD42020162626). Available from: https://www.crd. york.ac.uk/prospero/display_record.php?RecordID= 162626.

\section{Search strategy}

A comprehensive search was done in 10/10/2019 to find related literature on international online databases Web of Science, Scopus, EMBASE, Science Direct, PubMed/ Medline, EBSCO, CINAHL, Cochrane Library (Cochrane Database of Systematic Reviews - CDSR), and national online databases Barakat Knowledge Network System (http://health.barakatkns.com), Magiran (http://www. magiran.com/), Regional Information Center for Science and Technology (RICST) (http://en.ricest.ac.ir/), Scientific Information Database (SID) (http://www.sid.ir/), Civilica (https://www.civilica.com/), Iranian Research Institute for Information Science and Technology (IranDoc ((https://irandoc.ac.ir), Iranian National Library (http:// www.nlai.ir/) and the Google Scholar search engine. The search was carried out without limitation in time and language. Keywords were: "Metabolism, Inborn Errors" [Mesh], "Metabolic Diseases" [Mesh], "Amino Acid Metabolism, Inborn Errors" [Mesh], "Phenylketonurias" [Mesh] and "Iran" [Mesh].

The keywords were combined using boolean operator "AND" and "OR". An example of a combined search in the Pubmed database was as follows: (((c"Metabolism, Inborn Errors" [Mesh]) OR "Metabolic Diseases" [Mesh]) OR "Amino Acid Metabolism, Inborn Errors" [Mesh]) OR "Phenylketonurias" [Mesh]) AND "Iran" [Mesh].

Search keywords were regulated based on minor specifications and differences in the syntax rules of each database. Reference lists of all retrieved articles were manually reviewed to identify all potential studies.

\section{Inclusion and exclusion criteria}

PICO (Patient, Population, or Problem; Intervention, Prognostic Factor, or Exposure; Comparison or Intervention (if appropriate); Outcome) [36] for inclusion and exclusion criteria were determined as follows: Inclusion criteria were all epidemiological studies about the prevalence of PKU that have been peer-reviewed at least in the form of abstract. Exclusion criteria were: 1) duplicate studies; 2) sample size other than infants (over 28 days of age); 3) non-random sample size; 4) non-Iranian studies; 5) being irrelevant; 6) sample size smaller than 200 
participants; 7) participants with certain diseases (e.g. mental retardation and etc); 8) unknown diagnostic intervention; 9) poor quality qualitative evaluation; 10), case reports, review articles, and letters to the editor without quantitative data.

\section{Study selection}

The title and abstract of all identified documentations were screened. Then, we evaluated the full text of the articles according to the inclusion and exclusion criteria. Finally, the raised disagreements were discussed and resolved in the presence of all authors.

\section{Definitions}

Suspected cases of HPA were defined as Phe serum levels in primary NBS (in some sources $2 \mathrm{mg} / \mathrm{dl}$ and in some $4 \mathrm{mg} / \mathrm{dl}$ ), and PKU was diagnosed in suspected cases of HPA after confirmatory tests. In this study, PKU it relates to all degrees of HPA, and PKU was classified into two categories: 1 . Phe concentrations between ( 2 or 4 ) and 20 were considered as mild to moderate HPA, and 2. Phe concentrations above 20 were considered as classical PKU [4].

\section{Data extraction}

Data extracted by the authors included first author's name, email of the corresponding author or the first author, year of publication, region/province, year of study, sample size (total, boys and girls), data collection method, diagnostic criteria, prevalence for each variable (suspected HPA, PKU, and types of PKU), and finally the extracted data were entered into Excel software (XP professional edition; Microsoft, Redmond, Washington, USA).

For duplicate publications, we contacted the corresponding author or the first author to clarify the original publication, and if we did not receive a response, we selected the study with the largest number of participants for overlapping cases. We also contacted the corresponding author when the article data was incomplete or ambiguous and resolved the problem.

\section{Quality evaluation}

As all studies eventually included the prevalence, so the quality of the studies was evaluated using a checklist for cross-sectional/prevalence studies by the modified Newcastle-Ottawa Scale (NOS) [37]. The quality of articles was classified into three categories of low, medium and high. Scoring was considered $0-5,6-7$, and $8-9$, respectively, and studies with poor quality excluded.

\section{Statistical analysis}

Heterogeneity among studies was assessed by $\mathrm{I}^{2}$ and $\mathrm{Q}$ tests. Interpretation of heterogeneity based on $\mathrm{I}^{2}$ Index is as follows: less than 25\% (low heterogeneity), 25-49\% (moderate heterogeneity) and 50-75\% (considerable heterogeneity), and greater than 75\% (high heterogeneity), and $P$-value less than 0.10 is statistically significant [38, 39]. In cases of low heterogeneity, the fixed effects model was used, and in other cases, the random effects model was used for data integration. Girls-boys Odds Ratio (OR) was used to indicate the effect of gender on suspected HPA and PKU, using HPA and PKU positive cases in both genders and the total sample size. To explore the cause of heterogeneity, subgroup analysis was done based on the region and the province of study, and sensitivity analysis was used to measure the overall estimation power by omitting one study at a time. Mixedeffects meta-regression was used to investigate the association between continuous variables such as the effect of time of study on prevalence. Publication bias was assessed by a visual survey of the funnel plot for skewed distribution, and using the Begg and Egger's tests [40, 41]. All meta-analyses were performed using Comprehensive Meta-Analysis Software (CMA) ver. 2. $P<0.05$ was considered significant in all tests.

\section{Results}

Search results and the features of studies included the meta-analysis

Figure 1 shows the flowchart of the selection of studies. Systematic search on databases and references identified 2126 related papers. Subsequently, 421 duplicate articles and 1672 unrelated articles were removed by reviewing the title and abstract. Then, 56 studies were excluded after reviewing the full-text since they did not meet the eligibility criteria. Finally, 18 studies with 3,339,327 Iranian neonates were included (Fig. 1).

Studies by Abbaskhanian A. et al. [16], Motamedi N. et al. [18], and Ganji F. et al. [28] were considered as more than one study each since they reported information in more than one population. All studies had appropriate quality to enter the meta-analysis (Table 1 ).

\section{Prevalence of suspected hyperphenylalaninemia}

Heterogeneity was high for these studies $\left(\mathrm{I}^{2}=98.41 \%\right.$; $P<0.001)$. The prevalence of suspected HPA in 873,174 Iranian neonates was estimated to be $45.6 / 100,000$ (95\% CI: 23.9-87.1) (Fig. 2 a). The lowest prevalence was related to the study of Soori in 2016-2017 $(8.8 / 100,000)$ and the highest prevalence was related to the study of Abbaskhanian in 2017 (600.1/100,000).

\section{Prevalence of suspected hyperphenylalaninemia based on gender}

The prevalence of suspected HPA in 285,331 girls infants and 297,347 boys infants in Iran was estimated to be 38.0/100,000 (95\% CI: $15.1-95.5$ ) and 43.3/100,000 


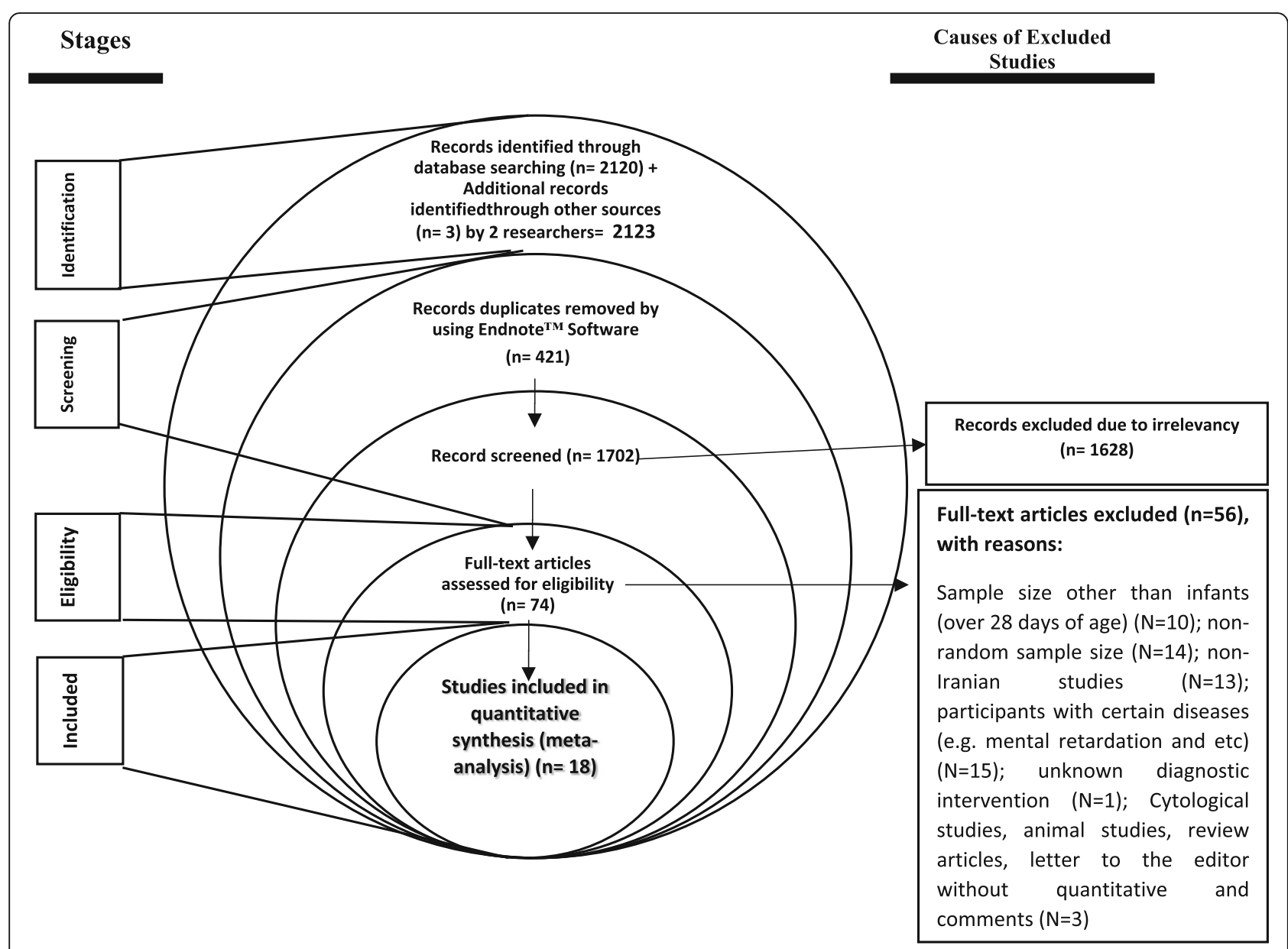

Fig. 1 PRISMA flowchart

(95\% CI: 16.2-116.2), respectively (Fig. 2 b-c). The girlsboys $\mathrm{OR}$ of suspected HPA was not significant $(\mathrm{OR}=$ 0.90 (95\% CI: 0.75-1.08; $P=0.259$ ) (Fig. 2-d).

\section{Subgroup analysis of the prevalence of suspected hyperphenylalaninemia}

Subgroup analysis of the prevalence of suspected HPA based on five geographical regions and provinces in Iran showed significant differences with $P<0.007$ and $P<$ 0.001 , respectively, but it was not significant in terms of the quality of studies $(P=0.241)$ (Fig. 3$)$.

\section{Prevalence of phenylketonuria}

Heterogeneity was high for the studies $\left(\mathrm{I}^{2}=82.56 \%\right.$; $P<$ 0.001). The prevalence of PKU in 3,000,917 Iranian neonates was estimated to be 16.5/100,000 (95\% CI: $12.9-$ 21.2). The lowest and highest prevalence was related to the studies of Abbaskhanian et al. (2.3/100,000) and Badiee et al. (144.3/100,000) (Fig. 4), respectively.
Prevalence of phenylketonuria based on gender

The prevalence of PKU in 285,331 girls and 297,347 boys infants was estimated to be $13.3 / 100,000$ (95\% CI: 9.319.0) and 10.9/100,000 (95\% CI: 7.5-15.8), respectively. The girls-boys OR of PKU prevalence was not significant $(\mathrm{OR}=1.58$ (95\% CI: 0.66-2.02, $P=0.606)$ (Fig. 5).

\section{Subgroup analysis of phenylketonuria prevalence}

The prevalence of PKU in West, East, North, South and Center of Iran was estimated to be 19.4/100,000 (95\% CI: 15.1-24.9), 31.9/100,000 (95\% CI: 9.0-113.3), 7.9/ 100,000 (95\% CI: 4.7-13.1), 17.2/100,000 (95\% CI: 13.621.8) and 21.3/100,000 (95\% CI: 10.2-44.6), respectively, and the differences in subgroup analysis were significant $(P<0.001)$. In subgroup analysis based on province, the lowest and highest prevalence of PKU was in Golestan $(5.4 / 100,000)$ and Isfahan $(58.4 / 100,000)$ provinces, respectively and the difference was significant $(P<0.001)$.

In subgroup analysis based on the quality of studies, the prevalence of PKU in medium and high-quality studies was estimated to be $22.2 / 100,000$ (95\% CI: 15.6-31.5) 


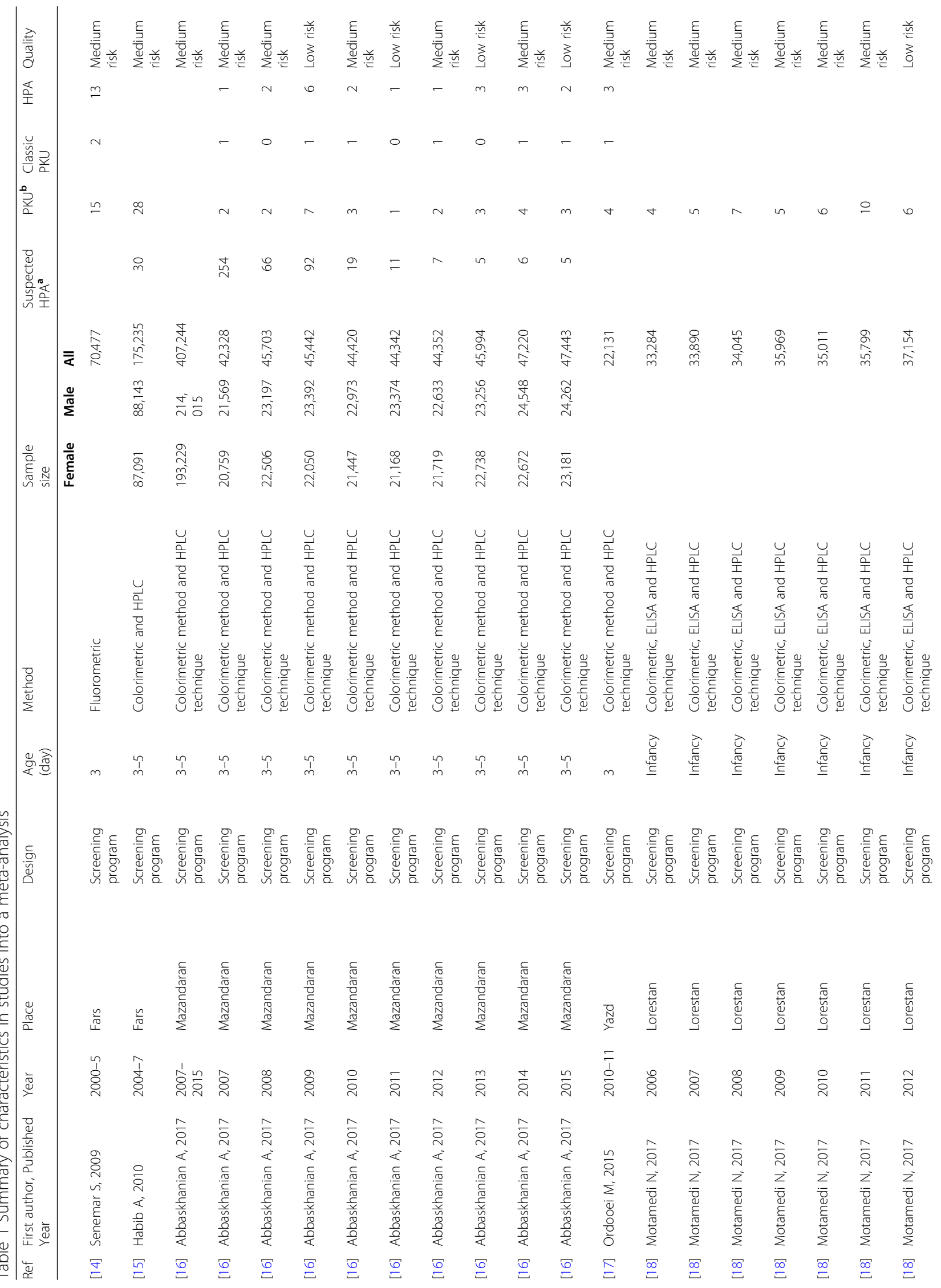




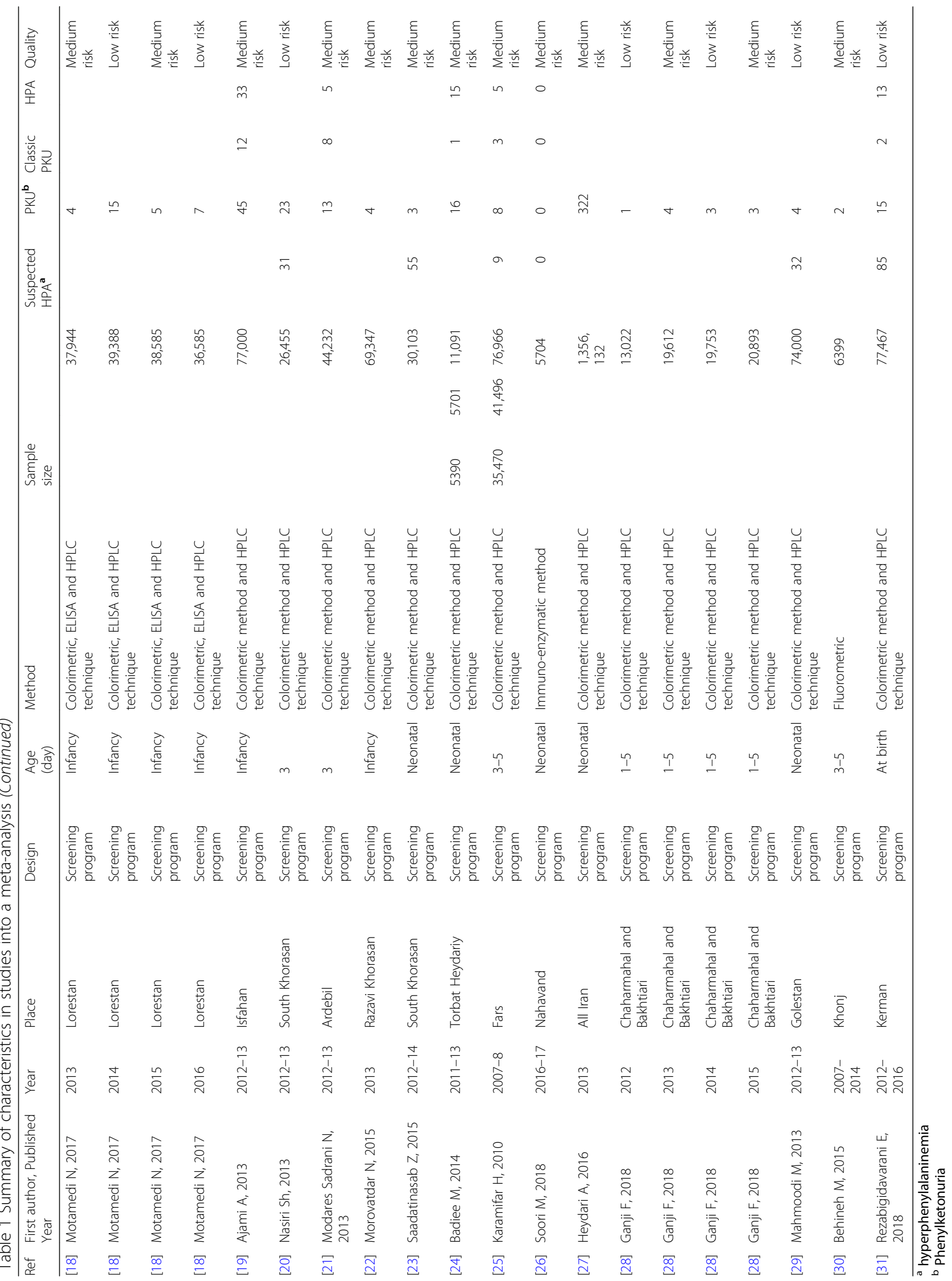




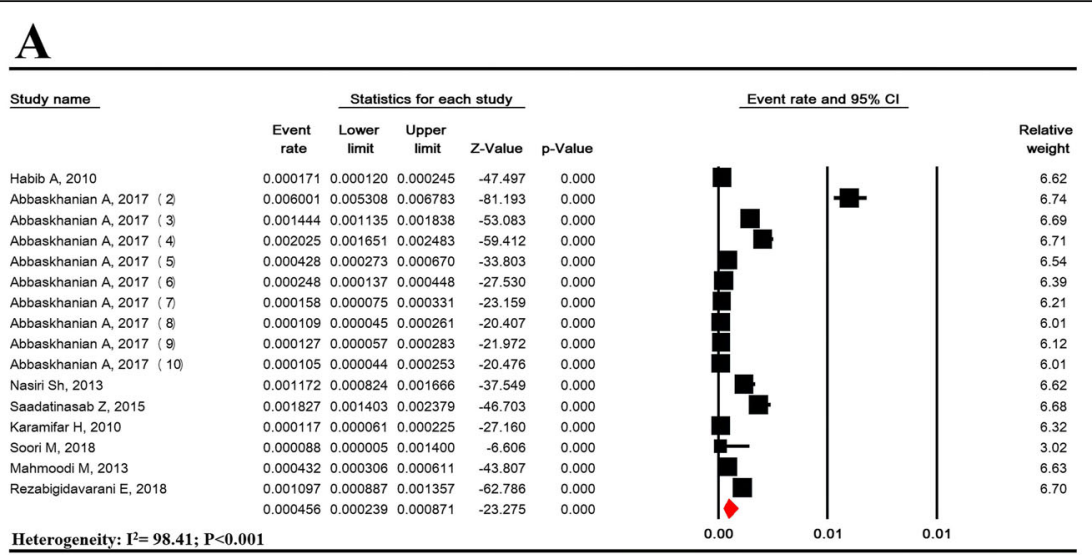

Heterogeneity: $\mathrm{I}^{2}=98.41 ; \mathrm{P}<0.001$

Meta Analysis

B

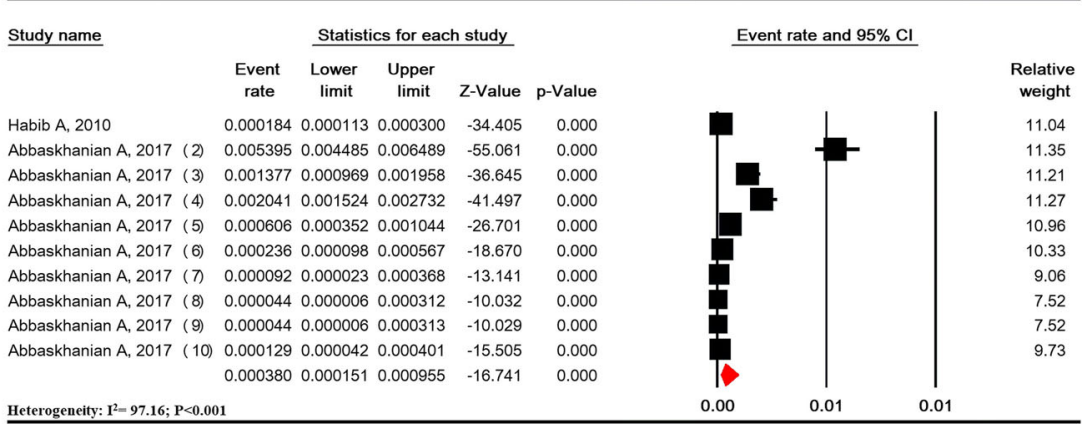

C

\begin{tabular}{|c|c|c|c|c|c|c|c|c|c|}
\hline \multirow[t]{2}{*}{ Study name } & \multirow[b]{2}{*}{$\begin{array}{c}\text { Event } \\
\text { rate }\end{array}$} & \multicolumn{3}{|c|}{ Statistics for each study } & \multicolumn{5}{|c|}{ Event rate and $95 \% \mathrm{Cl}$} \\
\hline & & $\begin{array}{l}\text { Lower } \\
\text { limit }\end{array}$ & $\begin{array}{c}\text { Upper } \\
\text { limit }\end{array}$ & Z-Value & $p$-Value & & & & $\begin{array}{c}\text { Relative } \\
\text { weight }\end{array}$ \\
\hline Habib A, 2010 & 0.000159 & 0.000094 & 0.000268 & -32.728 & 0.000 & & & & 10.34 \\
\hline Abbaskhanian A, 2017 (2) & 0.006584 & 0.005588 & 0.007756 & -59.582 & 0.000 & & -1 & & 10.62 \\
\hline Abbaskhanian A, 2017 (3) & 0.001509 & 0.001084 & 0.002101 & -38.395 & 0.000 & & & & 10.52 \\
\hline Abbaskhanian A, 2017 (4) & 0.002009 & 0.001510 & 0.002673 & -42.517 & 0.000 & & & & 10.55 \\
\hline Abbaskhanian A, 2017 (5) & 0.000261 & 0.000117 & 0.000581 & -20.206 & 0.000 & & & & 9.95 \\
\hline Abbaskhanian A, 2017 (6) & 0.000257 & 0.000115 & 0.000571 & -20.248 & 0.000 & & & & 9.95 \\
\hline Abbaskhanian A, 2017 (7) & 0.000221 & 0.000092 & 0.000531 & -18.820 & 0.000 & & & & 9.82 \\
\hline Abbaskhanian A, 2017 (8) & 0.000172 & 0.000065 & 0.000458 & -17.334 & 0.000 & & & & 9.63 \\
\hline Abbaskhanian A, 2017 (9) & 0.000204 & 0.000085 & 0.000489 & -19.002 & 0.000 & & & & 9.82 \\
\hline \multirow[t]{2}{*}{ Abbaskhanian A, 2017 (10) } & 0.000082 & 0.000021 & 0.000330 & -13.298 & 0.000 & & & & 8.80 \\
\hline & 0.000434 & 0.000162 & 0.001162 & -15.399 & 0.000 & & & & \\
\hline \multicolumn{2}{|l|}{ Heterogeneity: $I^{2}=97.88 ; P<0.001$} & & & & & 0.00 & 0.01 & 0.01 & \\
\hline
\end{tabular}

Meta Analysis

D

$\underline{\text { Study name }}$

Statistics for each study

Odds ratio and $95 \% \mathrm{Cl}$

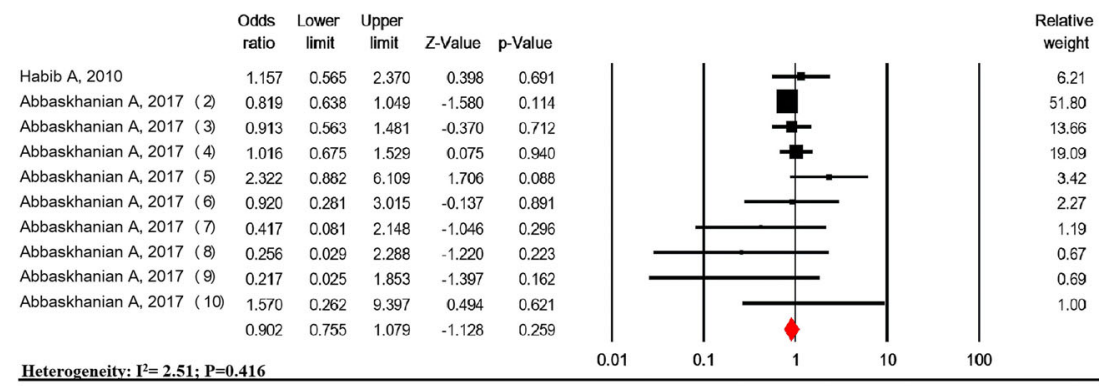

Heterogeneitv: $\mathrm{I}^{2}=2.51: \mathrm{P}=\mathbf{0 . 4 1 6}$

$\begin{array}{lllll}0.01 & 0.1 & 1 & 10 & 100\end{array}$

Fig. 2 Prevalence of suspected hyperphenylalaninemia in all (a), girls (b), and boys (c) and girls to boys odds ratio (d) in national neonate screening program in Iran 


\section{A}

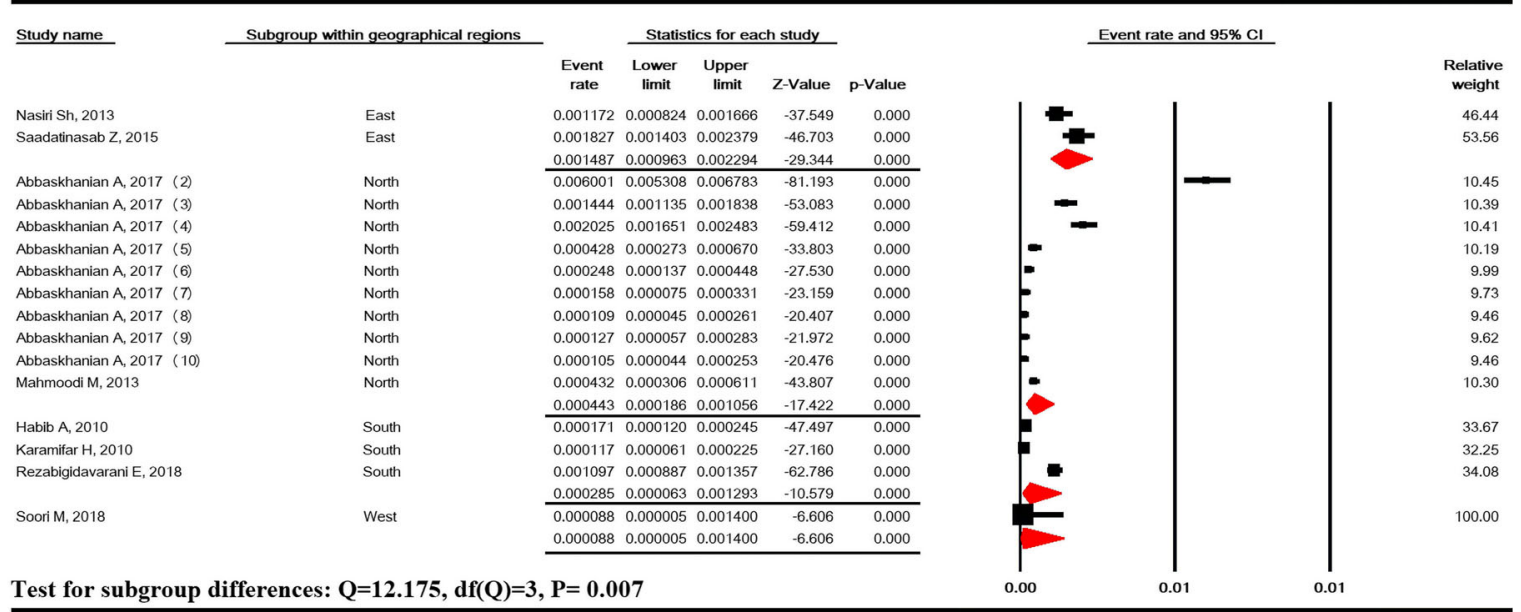

\section{B}

\begin{tabular}{|c|c|c|c|c|c|c|}
\hline \multirow[t]{2}{*}{ Study name } & \multirow[t]{2}{*}{ Subgroup within provinces } & \multirow[b]{2}{*}{$\begin{array}{c}\text { Event } \\
\text { rate }\end{array}$} & \multicolumn{3}{|c|}{ Statistics for each study } & \multirow[b]{2}{*}{$\mathrm{p}$-Value } \\
\hline & & & $\begin{array}{c}\text { Lower } \\
\text { limit }\end{array}$ & $\begin{array}{c}\text { Upper } \\
\text { limit }\end{array}$ & Z-Value & \\
\hline Habib A, 2010 & Fars & 0.000171 & 0.000120 & 0.000245 & -47.497 & 0.000 \\
\hline \multirow[t]{2}{*}{ Karamifar $\mathrm{H}, 2010$} & Fars & 0.000117 & 0.000061 & 0.000225 & -27.160 & 0.000 \\
\hline & & 0.000157 & 0.000114 & 0.000215 & -54.403 & 0.000 \\
\hline \multirow[t]{2}{*}{ Mahmoodi M, 2013} & Golestan & 0.000432 & 0.000306 & 0.000611 & -43.807 & 0.000 \\
\hline & & 0.000432 & 0.000306 & 0.000611 & -43.807 & 0.000 \\
\hline \multirow[t]{2}{*}{ Soori M, 2018} & Hamadan & 0.000088 & 0.000005 & 0.001400 & -6.606 & 0.000 \\
\hline & & 0.000088 & 0.000005 & 0.001400 & -6.606 & 0.000 \\
\hline \multirow[t]{2}{*}{ Rezabigidavarani E, 2018} & Kerman & 0.001097 & 0.000887 & 0.001357 & -62.786 & 0.000 \\
\hline & & 0.001097 & 0.000887 & 0.001357 & -62.786 & 0.000 \\
\hline Abbaskhanian A, 2017 (2) & Mazandaran & 0.006001 & 0.005308 & 0.006783 & -81.193 & 0.000 \\
\hline Abbaskhanian A, 2017 (3) & Mazandaran & 0.001444 & 0.001135 & 0.001838 & -53.083 & 0.000 \\
\hline Abbaskhanian A, 2017 (4) & Mazandaran & 0.002025 & 0.001651 & 0.002483 & -59.412 & 0.000 \\
\hline Abbaskhanian A, 2017 (5) & Mazandaran & 0.000428 & 0.000273 & 0.000670 & -33.803 & 0.000 \\
\hline Abbaskhanian A, 2017 (6) & Mazandaran & 0.000248 & 0.000137 & 0.000448 & -27.530 & 0.000 \\
\hline Abbaskhanian A, 2017 (7) & Mazandaran & 0.000158 & 0.000075 & 0.000331 & -23.159 & 0.000 \\
\hline Abbaskhanian A, 2017 (8) & Mazandaran & 0.000109 & 0.000045 & 0.000261 & -20.407 & 0.000 \\
\hline Abbaskhanian A, 2017 (9) & Mazandaran & 0.000127 & 0.000057 & 0.000283 & -21.972 & 0.000 \\
\hline \multirow[t]{2}{*}{ Abbaskhanian A, 2017 (10) } & Mazandaran & 0.000105 & 0.000044 & 0.000253 & -20.476 & 0.000 \\
\hline & & 0.000446 & 0.000182 & 0.001093 & -16.839 & 0.000 \\
\hline Nasiri Sh, 2013 & South Khorasan & 0.001172 & 0.000824 & 0.001666 & -37.549 & 0.000 \\
\hline \multirow[t]{2}{*}{ Saadatinasab Z, 2015} & South Khorasan & 0.001827 & 0.001403 & 0.002379 & -46.703 & 0.000 \\
\hline & & 0.001487 & 0.000963 & 0.002294 & -29.344 & 0.000 \\
\hline
\end{tabular}

Test for subgroup differences: $Q=\mathbf{1 2 2 . 8 7 4}, \operatorname{df}(Q)=5, P<0.001$

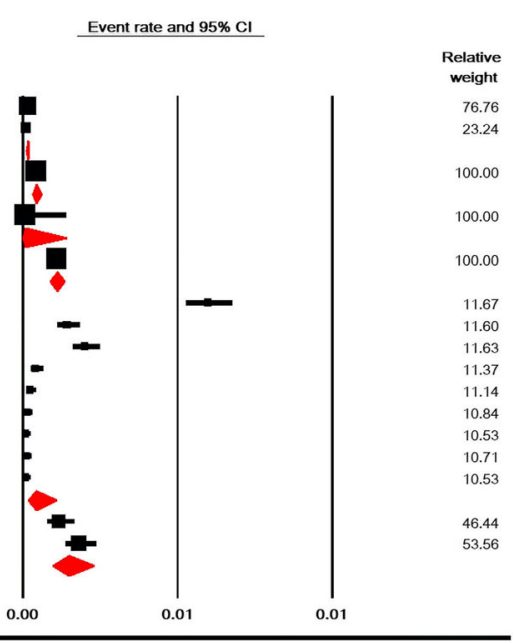

C

\begin{tabular}{|c|c|c|c|c|c|c|}
\hline \multirow[t]{2}{*}{ Study name } & \multirow[t]{2}{*}{$\underline{\text { Subgroup within quality of studies }}$} & \multicolumn{5}{|c|}{ Statistics for each study } \\
\hline & & $\begin{array}{c}\text { Event } \\
\text { rate }\end{array}$ & $\begin{array}{c}\text { Lower } \\
\text { limit }\end{array}$ & $\begin{array}{l}\text { Upper } \\
\text { limit }\end{array}$ & Z-Value & $\mathrm{p}$-Value \\
\hline Habib A, 2010 & High & 0.000171 & 0.000120 & 0.000245 & -47.497 & 0.000 \\
\hline Abbaskhanian A. 2017 (Z) & High & 0.006001 & 0.005308 & 0.006783 & -81.193 & 0.000 \\
\hline Abbaskhanian A, 2017 (3) & High & 0.001444 & 0.001135 & 0.001838 & -53.083 & 0.000 \\
\hline Abbaskhanian A, 2017 (4) & High & 0.002025 & 0.001651 & 0.002483 & -59.412 & 0.000 \\
\hline Abbaskhanian A 2017 (5) & High & 0.000428 & 0.000273 & 0.000670 & -33.803 & 0.000 \\
\hline Abbaskhanian A, 2017 (6) & High & 0.000248 & 0.000137 & 0.000448 & -27.530 & 0.000 \\
\hline Abbaskhanian A, 2017 (7) & High & 0.000158 & 0.000075 & 0.000331 & $-23,159$ & 0.000 \\
\hline Abbaskhanian A, 2017 (8) & High & 0.000109 & 0.000045 & 0.000261 & -20.407 & 0.000 \\
\hline Abbaskhanian A, 2017 (9) & High & 0.000127 & 0.000057 & 0.000283 & -21.972 & 0.000 \\
\hline Abbaskhanian A, 2017 (10) & High & 0.000105 & 0.000044 & 0.000253 & -20.476 & 0.000 \\
\hline Saadatinasab Z, 2015 & High & 0.001827 & 0.001403 & 0.002379 & -46.703 & 0.000 \\
\hline \multirow{2}{*}{ Karamifar $\mathrm{H}, 2010$} & High & 0.000117 & 0.000061 & 0.000225 & -27.160 & 0.000 \\
\hline & & 0.000414 & 0.000183 & 0.000937 & -18.667 & 0.000 \\
\hline Nasir Sh. 2013 & Moderate & 0.001172 & 0.000824 & 0.001666 & -37.549 & 0.000 \\
\hline Soori M. 2018 & Moderate & 0.000088 & 0.000005 & 0.001400 & -6.606 & 0.000 \\
\hline Mahmoodi M. 2013 & Moderate & 0.000432 & 0.000306 & 0.000611 & -43.807 & 0.000 \\
\hline Rezabigidavarani E, 2018 & Moderate & 0.001097 & 0.000887 & 0.001357 & -62.786 & 0.000 \\
\hline
\end{tabular}

Test for subgroup differences: $Q=1.374, \operatorname{df}(Q)=1, P=0.241$

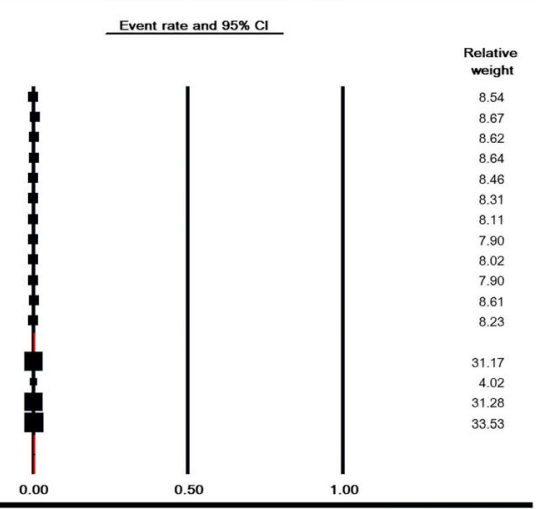
Meta Analysis

Fig. 3 Subgroup analysis of suspected hyperphenylalaninemia prevalence based on geographical regions (a), provinces (b) and the quality of studies (c) in national neonate screening program in Iran 


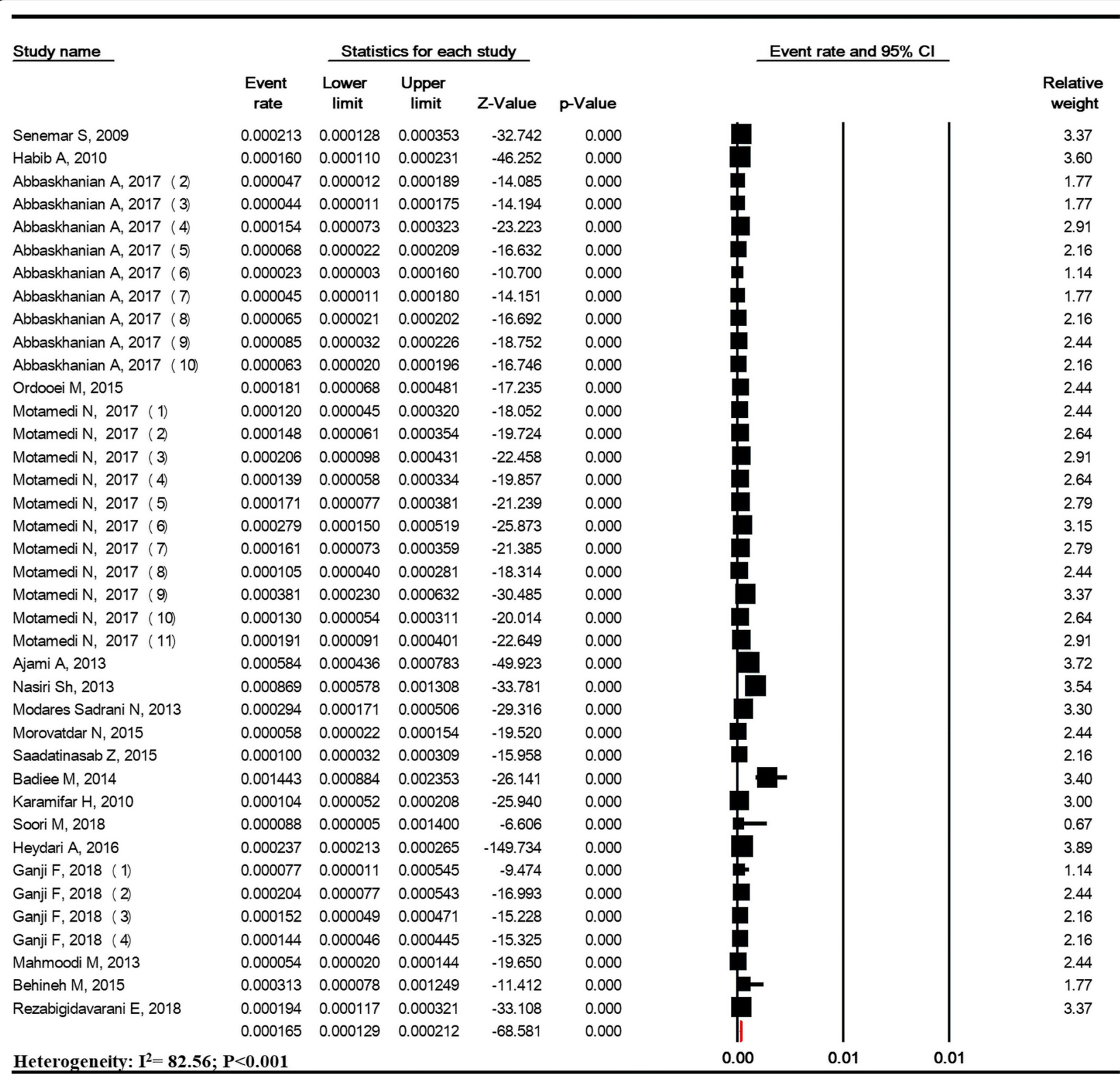

\section{Meta Analysis}

Fig. 4 Prevalence of phenylketonuria in national neonate screening program in Iran

and 10.1/100,000 (95\% CI: 7.1-14.5), respectively, and the differences in subgroup analysis were significant $(P=0.002)$ (Fig. 6).

\section{The prevalence of different types of phenylketonuria}

The prevalence of PKU types was investigated in 16 studies with a sample size of 714,845 Iranian neonates. The prevalence of mild to moderate HPA was estimated 9.7/100,000 (95\% CI: 5.1-18.4) and the prevalence of classical PKU was estimated 4.4/100,000 (95\% CI: 2.5-7.8)
(Fig. 7). In other words, the prevalence of mild to moderate HPA and the prevalence of classical PKU among PKU patients were $71.15 \%$ (95\% CI: 61.88-78.93) and 28.85\% (95\% CI: 21.07-38.12), respectively (Supplementary Figure 1).

\section{Meta-regression}

Meta-regression for the prevalence of suspected HPA, PKU, classical PKU and mild to moderate HPA based on the year of study was (meta-regression coefficient: $0.132,95 \%$ CI -0.346 to $0.081, P=0.226$ ), (meta- 


\section{A}

Study name

\begin{tabular}{|c|c|c|c|c|c|}
\hline & \multirow[b]{2}{*}{$\begin{array}{c}\text { Event } \\
\text { rate }\end{array}$} & & \multirow[b]{2}{*}{$\mathrm{p}$-Valc } \\
\hline & & $\begin{array}{c}\text { Lower } \\
\text { limit }\end{array}$ & $\begin{array}{c}\text { Upper } \\
\text { limit }\end{array}$ & Z-Value & \\
\hline Habib A, 2010 & 0.000172 & 0.000104 & 0.000286 & -33.562 & 0.6 \\
\hline Abbaskhanian A, 2017 (2) & 0.000096 & 0.000024 & 0.000385 & -13.077 & 0.0 \\
\hline Abbaskhanian A, 2017 (3) & 0.000022 & 0.000001 & 0.000355 & -7.576 & 0.0 \\
\hline Abbaskhanian A, 2017 (4) & 0.000227 & 0.000094 & 0.000545 & -18.762 & 0.0 \\
\hline Abbaskhanian A, 2017 (5) & 0.000140 & 0.000045 & 0.000434 & -15.370 & 0.0 \\
\hline Abbaskhanian A, 2017 (6) & 0.000047 & 0.000007 & 0.000335 & -9.960 & 0.0 \\
\hline Abbaskhanian A, 2017 (7) & 0.000046 & 0.000006 & 0.000327 & -9.986 & 0.6 \\
\hline Abbaskhanian A, 2017 (8) & 0.000022 & 0.000001 & 0.000351 & -7.584 & 0.0 \\
\hline Abbaskhanian A, 2017 (9) & 0.000044 & 0.000006 & 0.000313 & -10.029 & 0.0 \\
\hline Abbaskhanian A, 2017 (10) & 0.000043 & 0.000006 & 0.000306 & -10.051 & 0.0 \\
\hline & 0.000133 & 0.000093 & 0.000190 & -48.893 & \\
\hline
\end{tabular}

Event rate and $95 \% \mathrm{Cl}$

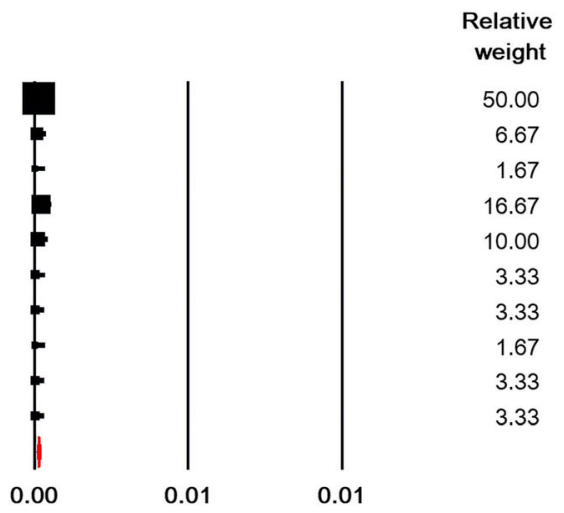

Heterogeneity: $\mathrm{I}^{2}=14.65 ; \mathrm{P}=0.308$

Meta Analysis

B

Study name

Statistics for each study

Event rate and $95 \% \mathrm{Cl}$

\begin{tabular}{|c|c|c|c|c|c|c|c|}
\hline & $\begin{array}{l}\text { Event } \\
\text { rate }\end{array}$ & $\begin{array}{c}\text { Lower } \\
\text { limit }\end{array}$ & $\begin{array}{c}\text { Upper } \\
\text { limit }\end{array}$ & Z-Value & p-Value & & $\begin{array}{c}\text { Relative } \\
\text { weight }\end{array}$ \\
\hline Habib A, 2010 & 0.000147 & 0.000086 & 0.000254 & -31.804 & 0.000 & & 47.27 \\
\hline Abbaskhanian A, 2017 & (2) 0.000023 & 0.000001 & 0.000370 & -7.546 & 0.000 & & 1.82 \\
\hline Abbaskhanian A, 2017 & (3) 0.000086 & 0.000022 & 0.000345 & -13.234 & 0.000 & & 7.27 \\
\hline Abbaskhanian A, 2017 & (4) 0.000085 & 0.000021 & 0.000342 & -13.246 & 0.000 & & 7.27 \\
\hline Abbaskhanian A, 2017 & (5) 0.000022 & 0.000001 & 0.000348 & -7.591 & 0.000 & & 1.82 \\
\hline Abbaskhanian A, 2017 & (6) 0.000021 & 0.000001 & 0.000342 & -7.603 & 0.000 & & 1.82 \\
\hline Abbaskhanian A, 2017 & (7) 0.000044 & 0.000006 & 0.000314 & -10.027 & 0.000 & & 3.64 \\
\hline Abbaskhanian A, 2017 & (8) 0.000129 & 0.000042 & 0.000400 & -15.511 & 0.000 & & 10.91 \\
\hline Abbaskhanian A, 2017 & (9) 0.000122 & 0.000039 & 0.000379 & -15.604 & 0.000 & & 10.91 \\
\hline \multirow[t]{2}{*}{ Abbaskhanian A, 2017} & (10)0.000082 & 0.000021 & 0.000330 & -13.298 & 0.000 & & 7.27 \\
\hline & 0.000109 & 0.000075 & 0.000158 & -47.850 & 0.000 & & \\
\hline \multicolumn{5}{|c|}{ Heterogeneity: $\mathrm{I}^{2}=0 ; \mathrm{P}=\mathbf{0 . 7 0 6}$} & 0.00 & 0.01 & \\
\hline
\end{tabular}

Meta Analvsis

$\underline{\mathrm{C}}$

Study name

Habib A, 2010

Abbaskhanian A, 2017 (2)

Abbaskhanian A, 2017 (3)

Abbaskhanian A, 2017 (4)

Abbaskhanian A, 2017 (5)

Abbaskhanian A, 2017 (6)

Abbaskhanian A, 2017 ( 7 )

Abbaskhanian A, 2017 (8)

Abbaskhanian A, 2017 (9)

Abbaskhanian A, 2017 (10)

Heterogeneity: $I^{2}=0 ; P=0.492$

Meta Analysis
Statistics for each study

Odds Lower Upper

ratio limit limit Z-Value $p$-Value

$\begin{array}{lllll}1.168 & 0.556 & 2.454 & 0.409 & 0.682\end{array}$

$\begin{array}{lllll}5.196 & 0.249 & 108.227 & 1.064 & 0.287\end{array}$

$\begin{array}{llllll}0.206 & 0.010 & 4.294 & -1.019 & 0.308\end{array}$

$\begin{array}{lllll}2.653 & 0.515 & 13.673 & 1.166 & 0.244\end{array}$

$\begin{array}{rrrrr}7.499 & 0.387 & 145.188 & 1.333 & 0.183\end{array}$

$\begin{array}{lllll}3.313 & 0.135 & 81.325 & 0.733 & 0.463\end{array}$

$\begin{array}{lllll}1.042 & 0.065 & 16.661 & 0.029 & 0.977\end{array}$

$\begin{array}{lllll}0.146 & 0.008 & 2.828 & -1.272 & 0.203\end{array}$

$\begin{array}{lllll}0.361 & 0.038 & 3.470 & -0.883 & 0.377\end{array}$

$\begin{array}{lllll}0.523 & 0.047 & 5.771 & -0.529 & 0.597\end{array}$

$\begin{array}{llll}0.663 & 2.020 & 0.516 & 0.606\end{array}$

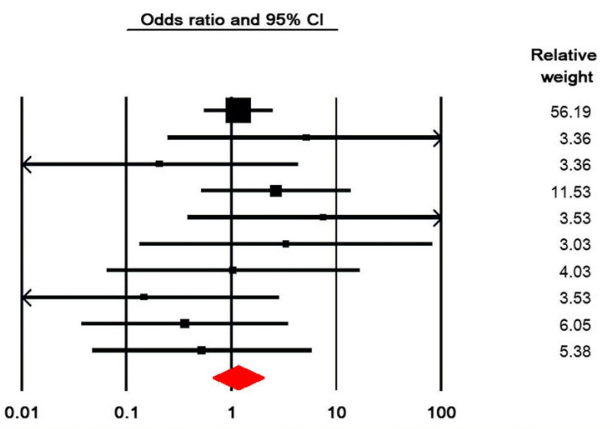

0.01

0.1

Fig. 5 Prevalence of phenylketonuria in girls (a) and boys (b) and girls to boys odds ratio (d) in national neonate screening program in Iran in Iran 

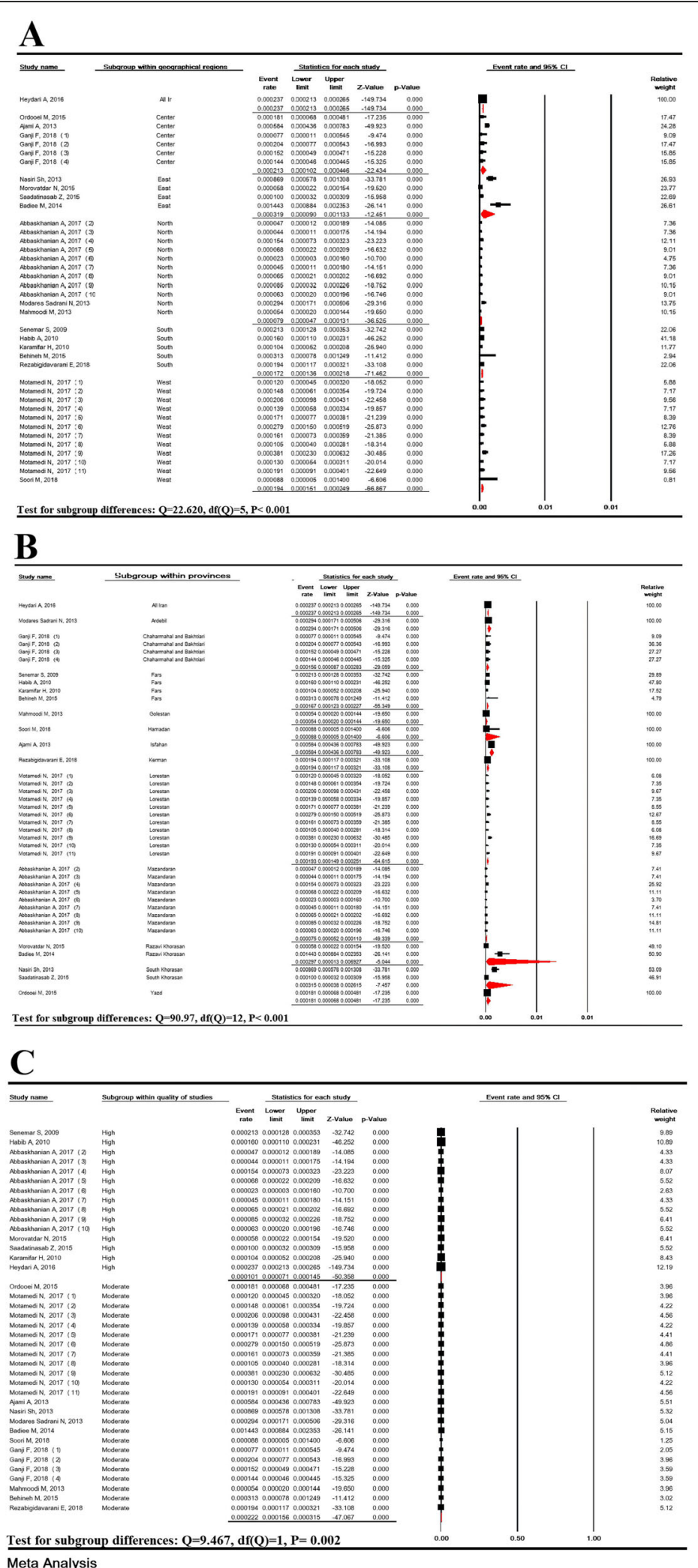

Fig. 6 Subgroup analysis of phenylketonuria prevalence based on geographical regions (a), provinces (b) and the quality of studies (c) in national neonate screening program in Iran 


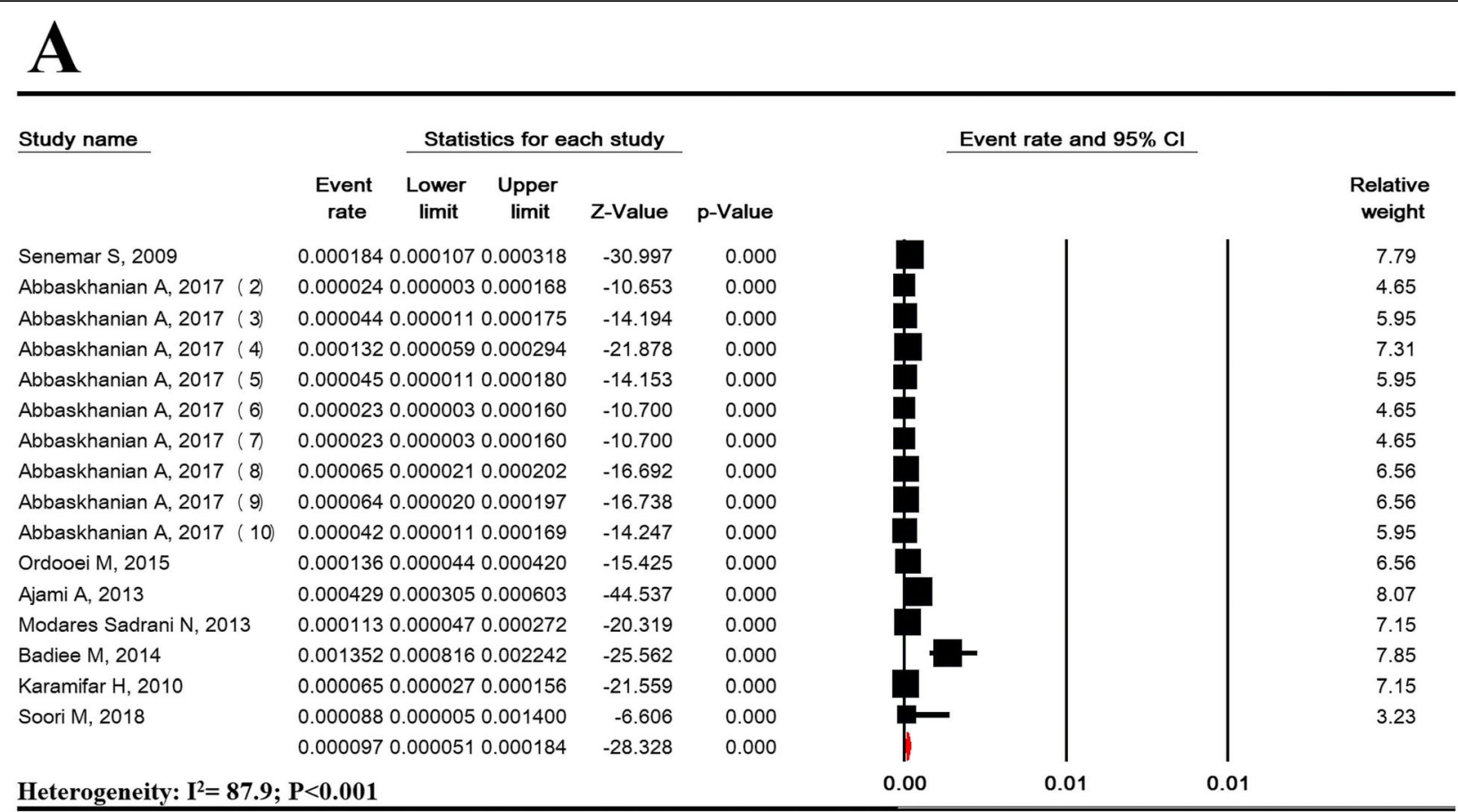

Meta Analysis

B

Study name

Senemar S, 2009
Abbaskhanian A, 2017 ( 2)
Abbaskhanian A, 2017 ( 3 )
Abbaskhanian A, 2017 (4)
Abbaskhanian A, 2017 (5)
Abbaskhanian A, 2017 ( 6 )
Abbaskhanian A, 2017 (7)
Abbaskhanian A, 2017 ( 8 ()
Abbaskhanian A, 2017 (9)
Abbaskhanian A, 2017 ( 10$)$
Ordooei M, 2015
Ajami A, 2013
Modares Sadrani N, 2013
Badiee M, 2014
Karamifar H, 2010
Soori M, 2018

\begin{tabular}{|c|c|c|c|c|}
\hline \multirow[b]{2}{*}{$\begin{array}{c}\text { Event } \\
\text { rate }\end{array}$} & \multicolumn{3}{|c|}{ Statistics for each study } & \multirow[b]{2}{*}{ p-Value } \\
\hline & $\begin{array}{l}\text { Lower } \\
\text { limit }\end{array}$ & $\begin{array}{l}\text { Upper } \\
\text { limit }\end{array}$ & Z-Value & \\
\hline 00028 & 0.000007 & 0.000113 & -14.806 & 0.000 \\
\hline 000024 & 0.000003 & 0.000168 & -10.653 & 0.000 \\
\hline 000011 & 0.000001 & 0.000175 & -8.077 & 0.000 \\
\hline 000022 & 0.000003 & 0.000156 & -10.724 & 0.000 \\
\hline .000023 & 0.000003 & 0.000160 & -10.701 & 0.000 \\
\hline .000011 & 0.000001 & 0.000180 & -8.056 & 0.000 \\
\hline .000023 & 0.000003 & 0.000160 & -10.700 & 0.000 \\
\hline .000011 & 0.000001 & 0.000174 & -8.082 & 0.000 \\
\hline .000021 & 0.000003 & 0.000150 & -10.762 & 0.000 \\
\hline .000021 & 0.000003 & 0.000150 & -10.767 & 0.000 \\
\hline 000045 & 0.000006 & 0.000321 & -10.004 & 0.000 \\
\hline 000156 & 0.000089 & 0.000274 & -30.366 & 0.000 \\
\hline .000181 & 0.000090 & 0.000362 & -24.372 & 0.000 \\
\hline 0090 & 0.000013 & 0.000640 & -9.313 & 0.000 \\
\hline 039 & 0.000013 & 0.000121 & -17.584 & 0.000 \\
\hline .000088 & 0.000005 & 0.001400 & -6.606 & 0.000 \\
\hline 0044 & 0.000025 & 0.000078 & -34.643 & 0.000 \\
\hline
\end{tabular}

Heterogeneity: $\mathrm{I}^{2}=50.80 ; \mathrm{P} 0.010$
Event rate and $95 \% \mathrm{Cl}$

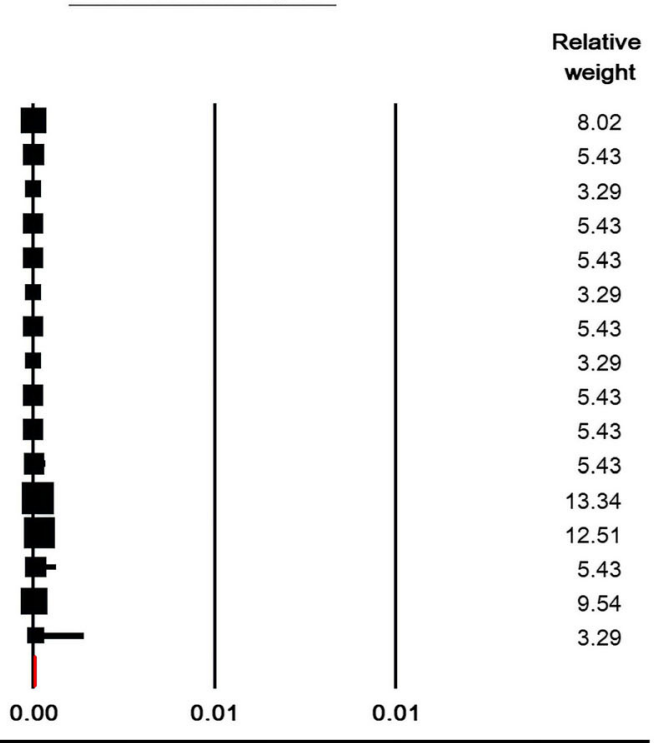

Meta Analysis

Fig. 7 Prevalence of mild to moderate hyperphenylalaninemia (a) classic phenylketonuria (b) in national neonate screening program in Iran

regression coefficient: $0.008,95 \% \mathrm{CI}-0.076$ to $0.92, P=$ 0.851), (meta-regression coefficient: $0.101,95 \%$ CI 0.213 to $0.416, P=0.528$ ) and (meta-regression coefficient: $0.020,95 \% \mathrm{CI}-0.212$ to $0.416, P=0.253)$, respectively (Supplementary Figure 2).
Sensitivity analysis and publication bias

Sensitivity analysis for prevalence of suspected HPA, PKU, classical PKU, and mild to moderate HPA with the omission of one study showed that overall estimation is still robust (Supplementary Figures 3, 4, 5). Egger's and 
Begg's tests for HPA prevalence $(P=0.137$ and $P<0.001$, respectively), overall PKU prevalence $(P=0.005$ and $P=$ 0.009), classical PKU $(P<0.001$ and $P=0.002)$ and mild to moderate PKU $(P=0.710$ and $P<0.001)$ were significant (Supplementary Figure 6).

\section{Discussion}

The present study is the first comprehensive systematic review and meta-analysis on PKU screening in Iranian newborns. The prevalence of PKU in Iranian neonates was estimated to be $16.5 / 100,000$. NBS is an important form of prevention in newborns with congenital metabolic diseases. This activity is very useful for detecting errors in many birth-related errors. It should be noted that many types of congenital disorders can be successfully treated if early diagnosis is achieved. If specific biochemical supplements are taken at an early stage, many metabolic disorders will be stopped from progressing and causing permanent damage to patients. The NBS is managed under the national public health policies. Metabolic disorders of the nervous system vary clinically and pathologically. Mental retardation and epilepsy syndrome are prominent in these disorders [42, 43].

The first pilot study to assess HPA in neonates in Iran began in 1982 [44] and the first National Neonate Screening Program (NNSP) in Iran started in Fars province in 2004 [45] and continued in Mazandaran province in 2007
[16]. Based on law, all Iranian infants should be screened for three diseases, including hypothyroidism, PKU, and glucose 6-phosphate dehydrogenase deficiency.

The published evidence confirms that the global NBS for PKU meets all accepted screening criteria and justifies the cost and infrastructure needed to collect and test dried blood spots [46]. Screening in infants is considered a national duty even in countries where there is no PKU population. Because of high migration in countries, detection of PKU has remained probable. Neonatal screening requires: 1) a solid infrastructure in which blood is collected from all neonates to ensure timely treatment; 2 ) a well-equipped laboratory that can effectively administer blood spot. Lowincome countries may consider using NBS laboratory facilities of other countries [47, 48].

In the present study, the prevalence of suspected HPA $(45.6 / 100,000)$ was much higher than the prevalence of PKU. Differential diagnosis of HPA includes high intake of natural protein, liver disease, tetrahydrobiopterin (BH4) deficiency, and being premature $[49,50]$.

The prevalence of PKU varies across ethnic groups and geographical regions around the world [51]. The prevalence of $\mathrm{PKU}$ has been reported to range from less than $1 / 220,000$ to $1 / 4500$. Table 2 summarizes the prevalence of PKU in different populations. In the present study, due to ethnic differences in different geographical regions of Iran, the prevalence of PKU

Table 2 Summarizes the prevalence of PKU in different populations

\begin{tabular}{|c|c|c|}
\hline Regions & Countries & Incidence of $\mathrm{PKU}^{\mathrm{a}}$ \\
\hline \multirow[t]{8}{*}{ Asian populations } & Turkey, 1986 (53) & 1: 4500 \\
\hline & Saudi Arabia, 2017 [8] & 1: 14245 \\
\hline & United Arab of Emirates, 2000 (54) & 1: 20050 \\
\hline & United Arab of Emirates, 2014 (55) & 1: 14544 \\
\hline & Iraq, 2015 (56) & 1.2: 10000 \\
\hline & Thailand 2009 and $2015(13,57)$ & $<1: 220,000$ \\
\hline & Mexico, 2012 (58) & $1: 161,748$ \\
\hline & Japan, 2017 (59) & 1:143,000 \\
\hline \multirow[t]{8}{*}{ European populations } & Ireland, 1978 (60) & 1 in 4500 \\
\hline & Sweden, before and after 1990 [52] & $1: 18,300$ to $1: 14,200$ \\
\hline & Germany, 2002 and 2014 (61) & $1: 10,339$ \\
\hline & Greece, 2016 (62) & 1: 10000 \\
\hline & Bulgaria, 2016 (62) & 1: 18000 \\
\hline & Poland, 2016 (62) & 1: 7000 \\
\hline & Spain, 2016 (62) & 1: 7400 \\
\hline & Italy, 2016 (62) & 1: 11500 \\
\hline South America & Brazil, 2014 (63) & $1: 8690$ \\
\hline \multirow[t]{2}{*}{ North America } & United States (Caucasians) (64) & 1 in 10,000 \\
\hline & Canada, 1986 (65) & 1 in 22,000 \\
\hline
\end{tabular}

\footnotetext{
${ }^{\text {a }}$ Phenylketonuria
} 
was significantly different in five different regions of Iran.

In the present study, prevalence of mild to moderate HPA and prevalence of classical PKU among PKU patients were 71.15 and $28.85 \%$, respectively, indicating that the majority of patients with PKU suffer from mild to moderate HPA in Iran. This can play a key role in the initiation and non-initiation of PKU treatment and management of these patients. That's because untreated Phe concentration determines the management of people with PKU. If blood Phe concentrations are below $360 \mu \mathrm{mol} / \mathrm{l}$, no intervention is required. If Phe blood concentration is between $360 \mu \mathrm{mol} / \mathrm{l}$ and $600 \mu \mathrm{mol} / \mathrm{l}$, treatment up to the age of 12 is recommended and lifelong treatment is recommended if the concentration is above $600 \mu \mathrm{mol} / \mathrm{l}$. For women trying to get pregnant (maternal PKU), untreated Phe blood concentration drops to more than $360 \mu \mathrm{mol} / \mathrm{l}$. On the other hand, management of PKU is associated with a severe financial burden on patients' families, which may lead to inadequate treatment or a change in blood Phe concentration $[2,3]$.

The trend of changes in the prevalence of suspected HPA, PKU, classical PKU and mild to moderate HPA did not change significantly over time. PKU as an autosomal-recessive disorder is not only related to consanguineous marriage and also occurs in regions with a low incidence of consanguineous marriage e.g. Europe [52]. In Iran, three main areas of prevention and control of hereditary metabolic diseases for PKU include NSB for this disease, selected one-stop clinics and premarriage screening program. Therefore, one can say that Iran's national programs regarding pre-marriage genetic counseling have not been effective in reducing PKU.

This study has several strengths: 1) A comprehensive search strategy was used in this study to maximize the possibility of identifying all relevant literature and even gray literature; 2) All research steps were conducted independently by two researchers, and disagreements were resolved by group discussion, 3) To obtain additional information and to make decisions about duplicate publication, we contacted the authors of the studies, 4) In cases where heterogeneity was significant, the random effects model was used to integrate data to provide a conservative estimate and on the other hand, subgroup analysis and meta-regression model were used to find the cause of heterogeneity and publication bias, and 5) We excluded studies on certain patients such as mentally retarded patients or studies with non-random sample and the resulting estimate can be generalized to the total population.

The limitations of the present study include the limitation of Iranian databases in combined search. In addition, there was a high heterogeneity among studies that investigated the prevalence of suspected HPA and PKU, and based on the available data, we were only able to do subgroup analysis based on geographical regions and provinces, which was significant. Therefore, the differences between studies can be attributed to these issues. However, it seems that more important issues such as differences in the percentage of consanguineous marriages and genetic differences between different populations of Iran (given that Iran includes various ethnic groups) may also be the reason for differences between studies. It was not possible to address these issues in this study. Other studies including the study of Hardelid et al. in England showed that the prevalence of PKU is lower among the Sub-Saharan Africans and South Asians who migrated to England [51]. Studies in other European countries showed that the increased incidence of PKU may be due to new mutations in this disease and migration of people of different races to their country [52].

\section{Conclusions}

The prevalence of PKU in Iran was estimated to be 16.6/ 100,000 or $1 / 6.024$. Due to ethnic and demographic similarities in Iran, we may also expand our results and estimates to Iranians living in other countries. It should be considered that for phenylketonuria there is a highly effective dietary treatment which can prevent the clinical symptoms of phenylketonuria if initiated early after detection by newborn screening.

\section{Supplementary information}

Supplementary information accompanies this paper at https://doi.org/10. 1186/s12887-020-02230-6.

Additional file 1: Figure 1. Prevalence of mild to moderate hyperphenylalaninemia (a) and classic phenylketonuria (b) among phenylketonuria patients.

Additional file 2: Figure 2. Meta-regression model for prevalence of suspected hyperphenylalaninemia (a), phenylketonuria (b), classic phenylketonuria (c), and mild to moderate hyperphenylalaninemia (d) based on year of study.

Additional file 3: Figure 3. Sensitivity analysis for prevalence of suspected hyperphenylalaninemia in national neonate screening program in Iran.

Additional file 4: Figure 4. Sensitivity analysis for prevalence of phenylketonuria in national neonate screening program in Iran.

Additional file 5: Figure 5. Sensitivity analysis for prevalence of mild to moderate hyperphenylalaninemia (a) classic phenylketonuria (b) in national neonate screening program in Iran.

Additional file 6: Figure 6. Publication bias for prevalence of suspected hyperphenylalaninemia (a), phenylketonuria (b), classic phenylketonuria (c), and mild to moderate hyperphenylalaninemia (d) based on year of study.

\section{Abbreviations}

PKU: Phenylketonuria; PAH: Phenylalanine hydroxylase; Phe: Phenylalanine; HPA: Hyperphenylalaninemia; NBS: Newborn screening; RICST: Regional 
Information Center for Science and Technology; SID: Scientific Information Database; IranDoc: Iranian Research Institute for Information Science and Technology; NNSP: National Neonate Screening Program; MOOSE: Metaanalyses Of Observational Studies in Epidemiology; PRISMA: Systematic Reviews and Meta-analysis; OR: Odds ratio; Cl: Confidence interval; DBS: Dried Blood Spot; HPLC: high-Performance Liquid Chromatographic; PROSPERO: International prospective register of systematic reviews; PICO: Patient, Population, or Problem; Intervention, Prognostic Factor, or Exposure; Comparison or Intervention (if appropriate); Outcome; NOS: Newcastle-Ottawa Scale; BH4: Tetrahydrobiopterin

\section{Acknowledgements}

We sincerely thank Ilam University of Medical Sciences for helping us with this research.

\section{Authors' contributions}

MA, MS, GhB, HZ, FK and PK acquired the data. MA and MS analyzed and interpreted the data. MS drafted the manuscript; MA, MS, GhB, HZ, FK and PK critically revised the manuscript for important intellectual content. M.S. supervised the study. All Authors read and approved the manuscript.

\section{Funding}

This study was funded by the llam University of Medical sciences. The role of the funding agency was only to financial support.

\section{Availability of data and materials}

Not applicable.

\section{Ethics approval and consent to participate}

Not applicable.

\section{Consent for publication}

Not applicable.

\section{Competing interests}

We declare no competing interests.

\section{Author details}

${ }^{1}$ Department of Pediatrics, School of Medicine, Ilam University of Medical Sciences, Ilam, Iran. ${ }^{2}$ School of Nursing and Midwifery, Ahvaz Jundishapour University of Medical Sciences, Ahvaz, Iran. ${ }^{3}$ School of Medicine, Qazvin University of Medical Sciences, Qazvin, Iran. ${ }^{4}$ Department of Pediatric, Faculty of Medicine, Ahvaz Jundishapour University of Medical Sciences, Ahvaz, Iran.

${ }^{5}$ School of Medicine, Ilam University of Medical Sciences, Ilam, Iran.

Received: 8 January 2020 Accepted: 30 June 2020

Published online: 24 July 2020

\section{References}

1. Mitchell JJ, Trakadis YJ, Scriver CR. Phenylalanine hydroxylase deficiency. Genet Med. 2011;13(8):697-707.

2. van Spronsen FJ, van Wegberg AM, Ahring K, Bélanger-Quintana A, Blau N, Bosch AM, et al. Key European guidelines for the diagnosis and management of patients with phenylketonuria. Lancet Diabetes Endocrinol. 2017;5(9):743-56.

3. Van Wegberg A, MacDonald A, Ahring K, Bélanger-Quintana A, Blau N, Bosch A, et al. The complete European guidelines on phenylketonuria: diagnosis and treatment. Orphanet J Rare Dis. 2017;12(1):162.

4. Mitchell JJ, Trakadis YJ, Scriver CR. Phenylalanine hydroxylase deficiency. Genet Med. 2011;13(8):697.

5. Blau N, Bélanger-Quintana A, Demirkol M, Feillet F, Giovannini M, MacDonald A, et al. Management of phenylketonuria in Europe: survey results from 19 countries. Mol Genet Metab. 2010;99(2):109-15.

6. Guthrie R, Susi A. A simple phenylalanine method for detecting phenylketonuria in large populations of newborn infants. Pediatrics. 1963; 32(3):338-43.

7. McCabe LL, Therrell BL Jr, McCabe ER. Newborn screening: rationale for a comprehensive, fully integrated public health system. Mol Genet Metab. 2002;77(4):267-73.
8. Alfadhel M, Al Othaim A, Al Saif S, Al Mutairi F, Alsayed M, Rahbeeni Z, et al. Expanded newborn screening program in Saudi Arabia: incidence of screened disorders. J Paediatr Child Health. 2017;53(6):585-91.

9. Yamaguchi A, Mizushima Y, Fukushi M, Shimizu Y, Kikuchi Y, Takasugi N. Microassay system for newborn screening for phenylketonuria, maple syrup urine disease, homocystinuria, histidinemia and galactosemia with use of a fluorometric microplate reader. Screening. 1992;1(1):49-62.

10. Contreras J, Alonso E, Fuentes LE. HPLC for confirmatory diagnosis and biochemical monitoring of Cuban patients with hyperphenylalaninemias. Medicc Rev. 2015;17:23-8.

11. Elvers L, Diependaal G, Blonk H, Loeber J. Phenylketonuria screening using the Quantase phenylalanine kit in combination with a microfilter system and the dye Tartrazine. Screening. 1995;3(4):209-23.

12. Schulze A, Kohlmueller D, Mayatepek E. Sensitivity of electrospray-tandem mass spectrometry using the phenylalanine/tyrosine-ratio for differential diagnosis of hyperphenylalaninemia in neonates. Clin Chim Acta. 1999; 283(1-2):15-20.

13. Thiboonboon $K$, Leelahavarong $P$, Wattanasirichaigoon D, Vatanavicharn $N$, Wasant $P$, Shotelersuk $V$, et al. An economic evaluation of neonatal screening for inborn errors of metabolism using tandem mass spectrometry in Thailand. PLoS One. 2015;10(8):e0134782.

14. Senemar S, Ganjekarimi H, Fathzadeh M, Tarami B, Bazrgar M. Epidemiological and clinical study of phenylketonuria (PKU) disease in the National Screening Program of neonates, Fars province, southern Iran. Iran J Public Health. 2009;38(2):58-64.

15. Habib A, Fallahzadeh MH, Kazeroni HR, Ganjkarimi AH. Incidence of phenylketonuria in southern Iran. Iran J Med Sci. 2010;35(2):137-9.

16. Abbaskhanian A, Zamanfar D, Afshar P, Asadpoor E, Rouhanizadeh $H_{\text {, }}$ Jafarnia A, et al. Incidence of neonatal Hyperphenylalaninemia based on high-performance liquid chromatography confirmatory technique in Mazandaran Province, northern Iran (2007-2015). Int J Prev Med. 2017:8:93.

17. Ordooei M, Jafarizadeh M, Mirzaei M, Ashoori H, Zare A, Shojaeifar H. Prevalence of neonatal hyperphenylalaninemia in Yazd Province, Iran. Iran J Med Sci. 2015;40(3):292-3.

18. Motamedi N, Goodarzi E, Pordanjani SR, Valizadeh R, Moradi Y, Sohrabivafa $\mathrm{M}$, et al. Incidence of phenylketonuria in Lorestan province, west of Iran (2006-2016). Int J Pediatrics-Mashhad. 2017;5:4713-21.

19. Ajami AMF, Lamuchi P, Koohi A. Assessment of the prevalence of of phenylketonuria and its different forms in Isfahan province. J Lab Diagn. 2013;5(22):P896

20. Nasiri S, KHOSRAVI H. Phenylketonuria disease prevalence in children of South Khorasan Province from September 2013 to November 2014. J Lab Diagn. 2013;5(22):P123.

21. Sadrani NM, Zaifizadeh M, Hazrati S, Mohammadzadeh MSS. Prevalence of Phenylketonuria in Newborns in Ardebil by Screening and Analyzing the Results. Tehran: The 8th International \& 13th National Congress on Quality Improvement in Clinical Laboratories; 2013.

22. Morovatdar N, Aval SB, Yazdi SMRH, Norouzi F, Mina T. The epidemiological and clinical study of phenylketonuria (PKU) patients in Khorasan, Northeastern Iran. Iran J Neonatol. 2015;6(1):19.

23. Saadatinasab Z, Sharifzadeh G. Incidence of phenylketonuria in southern Khorasan (2012-2014). J Birjand Univ Med Sci. 2015;22(3):286-92.

24. Badiee M, Kameli M, Sharifi S, Hosaini F. Prevalence of congenital phenylketonuria in neonates born in Torbate Heydarieh during 2011-2013. J Univ Med Sci Torbate Heydarieh. 2014;2(1):28-34.

25. Karamifar $\mathrm{H}$, Ordoei $\mathrm{M}$, Karamizadeh $\mathrm{Z}$, Amirhakimi $\mathrm{GH}$. Incidence of neonatal hyperphenylalaninemia in Fars province, South Iran. Iran J Pediatr. 2010;20(2):216.

26. Soori MBN, Sharifi A, Selgi M. Prevalence of neonatal phenylketonuria in Nahavand city in 2015-2016 and evaluation of the importance of phenylketonuria screening in neonates. Sari: The 3rd National Conference on Biology at Payam Noor University; 2018.

27. Heydari A, Samavat A, Haj Valizadeh F, Mansoura L. The incidence of phenylketonuria in Iran in 2013. Tehran: The 9th University Student Conference on Innovations in Health Sciences; 2016.

28. Ganji F, Naseri H, Rostampour N, Sedighi M, Lotfizadeh M. Assessing the phenylketonuria screening program in newborns, Iran 2015-2016. Acta Med Iran. 2018;56(1):49-55.

29. Mahmoodi M, Nasrabadi AG, Rajabloo A, Khatti T. Assessment of the Prevalence of Phenylketonuria and its Diffrent Forms in Golestan 
Province. Tehran: The 8th International \& 13th National Congress on Quality Improvement in Clinical Laboratories; 2015.

30. Behineh M, Jafari M, Pirouzi A, Rasti G, Pashangpour Z. The Prevalence of Phenylketonuria (PKU) in Infants Referred to the Health Center of Khonj. Tehran: The 8th International \& 13th National Congress on Quality Improvement in Clinical Laboratories; 2013.

31. Rezabigidavarani EKN, Arefi S, Faryabi R. Investigation of phenylketonuria incidence in Kerman city during 2012-2013. Tehran: The 11th University Student Conference on Innovation in Health Sciences; 2018.

32. Tavan H, Mohammadi I, Carson KV, Sayehmiri K, Sayehmiri F. Prevalence of Epilepsy in Iran: A Meta-Analysis and Systematic Review. Iran J Child Neurol. 2014;8(4):9-17.

33. Karimian M, Salamati M, Milad Azami M. The relationship between metabolic syndrome and increased risk of Barrett's esophagus: an updated systematic review and meta-analysis. BMC Gastroenterology. 2020;20(138).

34. Shamseer L, Moher D, Clarke M, Ghersi D, Liberati A, Petticrew M, et al. Preferred reporting items for systematic review and meta-analysis protocols (PRISMA-P) 2015: elaboration and explanation. Bmj. 2015;349:g7647.

35. Stroup DF, Berlin JA, Morton SC, Olkin I, Williamson GD, Rennie D, et al. Meta-analysis of observational studies in epidemiology: a proposal for reporting. Jama. 2000;283(15):2008-12.

36. Leonardo R. PICO: model for clinical questions. Evid Based Med Pract. 2018; 3(115):2.

37. Peterson J, Welch V, Losos M, Tugwell P. The Newcastle-Ottawa scale (NOS) for assessing the quality of nonrandomised studies in meta-analyses. Ottawa: Ottawa Hospital Research Institute; 2011.

38. Higgins P, Green S. Cochrane Handbook for Systematic Reviews of Interventions (Version 5.1. 0) Available from: http://handbook. cochrane. org/front_page. htm. Accessed on December 4th. 2015;2011.

39. Ades A, Lu G, Higgins J. The interpretation of random-effects meta-analysis in decision models. Med Decis Mak. 2005;25(6):646-54.

40. Begg CB, Mazumdar M. Operating characteristics of a rank correlation test for publication bias. Biometrics. 1994:50:1088-101.

41. Egger M, Smith GD, Schneider M, Minder C. Bias in meta-analysis detected by a simple, graphical test. Bmj. 1997;315(7109):629-34.

42. Sutivijit $Y$, Banpavichit $A$, Wiwanitkit $V$. Prevalence of neonatal hypothyroidism and phenylketonuria in southern Thailand: a 10-year report. Indian J Endocrinol Metab. 2011;15(2):115.

43. Pourfarzam M, Zadhoush F. Newborn screening for inherited metabolic disorders; news and views. J Res Med Sci. 2013;18(9):801.

44. Farhud DD, Kabiri M. Incidence of phenylketonuria (PKU) in Iran. Indian J Pediatr. 1982:49(400):685-8

45. Golipoor Z, Kashani IR, Akbari M, Mahmoudi R, Abbasi M, Nekoonam S. Incidence of Phenylketonuria in Southern Iran. Iran J Med Sci. 2010;35(2):37-9.

46. Geelhoed E, Lewis B, Hounsome D, O'Leary P. Economic evaluation of neonatal screening for phenylketonuria and congenital hypothyroidism. J Paediatr Child Health. 2005;41(11):575-9.

47. Lord J, Thomason MJ, Littlejohns P, Chalmers RA, Bain MD, Addison GM, et al. Secondary analysis of economic data: a review of cost-benefit studies of neonatal screening for phenylketonuria. J Epidemiol Community Health. 1999;53(3):179-86.

48. Thomason MJ, Lord J, Bain MD, Chalmers RA, Littlejohns P, Addison GM, et al. A systematic review of evidence for the appropriateness of neonatal screening programmes for inborn errors of metabolism. J Public Health. 1998;20(3):331-43.

49. Dhondt JL. Strategy for the screening of tetrahydrobiopterin deficiency among hyperphenylalaninaemic patients: 15-years experience. J Inherit Metab Dis. 1991;14(2):117-27.

50. Opladen T, Hoffmann GF, Blau N. An international survey of patients with tetrahydrobiopterin deficiencies presenting with hyperphenylalaninaemia. J Inherit Metab Dis. 2012:35(6):963-73.

51. Hardelid P, Cortina-Borja M, Munro A, Jones H, Cleary M, Champion M, et al. The birth prevalence of PKU in populations of European, south Asian and sub-Saharan African ancestry living in south East England. Ann Hum Genet. 2008;72(1):65-71

52. Ohlsson A. Neonatal screening in Sweden and disease-causing variants in phenylketonuria, galactosaemia and biotinidase deficiency; 2016.

\section{Publisher's Note}

Springer Nature remains neutral with regard to jurisdictional claims in published maps and institutional affiliations.

\section{Ready to submit your research? Choose BMC and benefit from}

- fast, convenient online submission

- thorough peer review by experienced researchers in your field

- rapid publication on acceptance

- support for research data, including large and complex data types

- gold Open Access which fosters wider collaboration and increased citations

- maximum visibility for your research: over $100 \mathrm{M}$ website views per year

At BMC, research is always in progress.

Learn more biomedcentral.com/submissions 